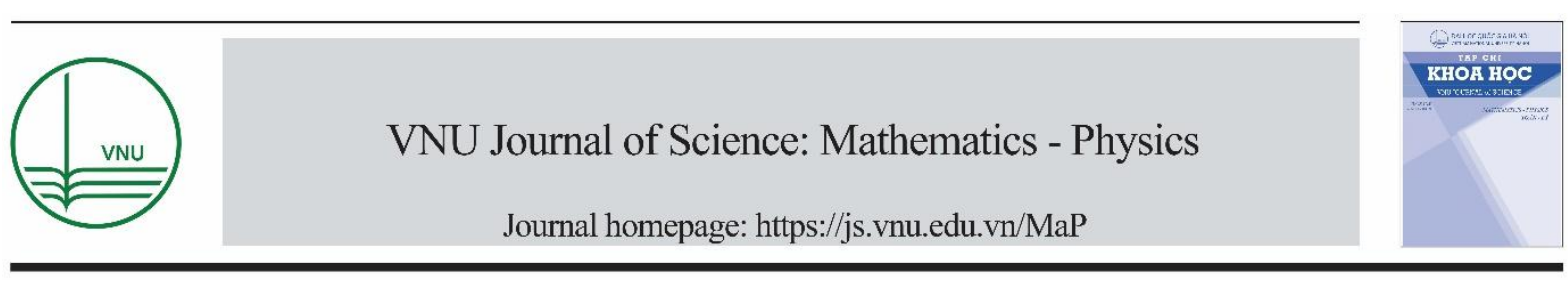

Original Article

\title{
Nonlinear Dynamic Response of Nano-composite Sandwich Annular Spherical Shells
}

\author{
Vu Thi Thuy Anh ${ }^{1}$, Vu Thi Huong ${ }^{1}$, Pham Dinh Nguyen ${ }^{1}$, \\ Nguyen Dinh Duc ${ }^{1,2, *}$, Vu Dinh Quang ${ }^{1,2}$ \\ ${ }^{1}$ Advanced Materials and Structures Laboratory, VNU Hanoi, University of Engineering and Technology \\ (UET), 144 Xuan Thuy, Cau Giay, Hanoi, Vietnam \\ ${ }^{2}$ Infrastructure Engineering Program, VNU Vietnam - Japan University (VJU), \\ My Dinh 1, Tu Liem, Hanoi, Vietnam
}

Received 10 July 2019

Accepted 05 August 2019

\begin{abstract}
This research considers the nonlinear dynamic response of functionally graded carbon nanotube reinforced composite (FG-CNTRC) sandwich annular spherical shells supported by Pasternak foundation by using the analytical approach. Unlike those discussed in previous studies, the composite considered in this study has three layers: FG-CNTRC layer - homogeneous core FG-CNTRC layer. Several examples are considered to analyse the behaviour of this sandwichstructured composite. The classical shell theory (CST) is used to derive theoretical formulation delineating nonlinear dynamic response of FG-CNTRC sandwich annular spherical shells. The numerical results explain the effect of material, geometrical parameters, and elastic foundations on the nonlinear dynamic response of the annular spherical shell.
\end{abstract}

Keywords: Nonlinear dynamic response, annular spherical shell, sandwich structure, FG-CNTRC.

\section{Introduction}

Carbon nanotubes (CNTs) were discovered in 1991 by Sumio Iijima [1] with many precious advantages such as high electrical and thermal conductivity, very high tensile strength, highly flexible and elastic, high aspect ratio, goof field emission. Therefore, CNTs are applied to the advanced materials as reinforcement components of composites in order to enhance structures' mechanical

\footnotetext{
${ }^{*}$ Corresponding author.

Email address: ducnd@ vnu.edu.vn
}

https//doi.org/ 10.25073/2588-1124/vnumap.4360 
properties. There are many publications that studied the static and dynamic behavior for CNTs reinforced composite plates and shells. Shen reported that the load-bending moment curves can be increased by CNT reinforcement in [2]. Farzam and Hassani [3] researched the thermal buckling and mechanical buckling of FG-CNTRC plate using isogeometric analysis. Trang and Tung [4] investigated the static behaviour of CNTRC cylindrical panels using the analytical approach. Zhong et al. [5] analysed the vibration of FG-CNTRC plate that are circular, annular and sector with arbitrary boundary conditions. The behaviour of FG-CNTRC plate and shell structures with forced vibration was studied by Frikha [6]. The nonlinear dynamic response and vibration of FG-CNTRC plates using third-order shear deformation was analysed by Thanh et al. [7].

The sandwich-structures are more attention to researchers and engineers due to their outstanding properties such as incredible bending rigidity, lightweight, excellent vibration characteristics. These sandwich-structures are made up of a moderately thick homogeneous core connected to thin FGM or nano-composite top and bottom layers. The sandwich configuration takes advantage of precious mechanical properties of top and bottom layers that are composed of super-stiffer materials. Dastjerdi and Aghadavoudi [8] analysed static behaviour of nano-composite sandwich plates which is reinforced by three types of defected carbon nanotubes using first order shear deformation theory. Wang and Shen [9] presented the examination of CNT's volume fraction and thermal loads on the nonlinear free vibration and bending behavior analysis of a sandwich plate placed on elastic foundation with top and bottom layers reinforced CNTs. Then, Shen et al. [10] developed this problem to analyze the nonlinear vibration of sandwich plates using the HSDT and von Kármán's assumption. Jalali and Heshmati [11] studied the buckling behavior of circular sandwich plates with three layers (tapered cores and nanocomposite top and bottom layers).

The annular spherical shells are widely applied in many engineering fields such as civil, mechanical, aerospace engineering. Alwar and Narasimhan [12] studied the nonlinear static behaviour of laminated orthotropic annular spherical shells. Dumir et al. [13] investigated the axisymmetric buckling behavior of laminated shallow annular spherical cap with various boundary conditions. The results of fundamental frequencies of the annular spherical shells are presented by Li et al. [14]. Duc et al. [15-16] presented nonlinear static response analysis of FGM annular spherical shells based on CST.

In the literature, there are many publications that studied the behaviour of many structures which made from functionally graded carbon nano-tube reinforced composite, but there isn't publication that research nonlinear dynamic response of FG-CNTRCs sandwich annular spherical shell on elastic foundation subjected to thermal and mechanical loads. This paper presents the nonlinear dynamic response of the nano-composite sandwich annular spherical shell supported by elastic foundation. The advantages of this work are applied the sandwich-structured configuration with three layers (top and bottom layers FG-CNTRC and a homogeneous core) to improve the mechanical properties of the annular spherical shell. A form of stress function for CNTs reinforced nano-composite annular spherical shells are presented. The governing equations of the CNTRC sandwich annular spherical shell are obtained by using the CST and von Kármán's geometrical nonlinearity.

\section{Material properties and formulation}

\subsection{Sandwich annular spherical shells}

Consider an annular spherical shell resting on elastic foundations with the radius of curvature $R$, base radii $r_{1}, r_{0}$ and thickness $h$. The shell is subjected to external pressure $q$ uniformly distributed on the outer surface as shown in Fig.1. The shell is defined in the coordinate system $(\varphi, \theta, \mathrm{z})$, where $\varphi$ 
and $\theta$ are in the meridional and circumferential direction of the shells, respectively and $z$ is perpendicular to the middle surface positive inwards. For a thin annular spherical shell, it is convenient to introduce a variable $r$, referred to as the radius of parallel circle with the base of shell and defined by $r=R \sin \varphi$. Moreover, due to the shallowness of the shell, it is approximately assumed that $\cos \varphi=1, R d \varphi=d r$.

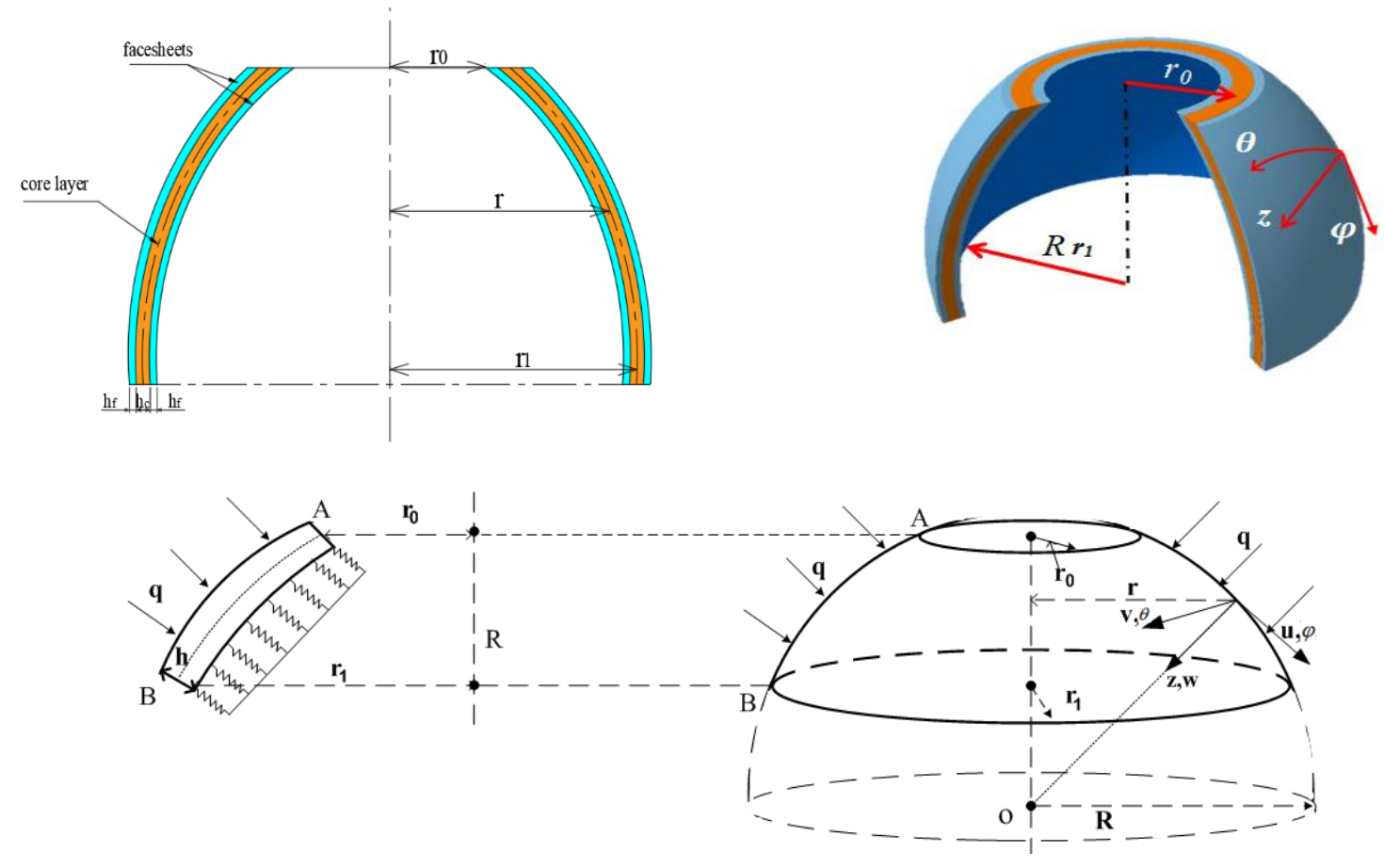

Fig. 1. Configuration of a sandwich nano-composite annular spherical shell.

In this present study, the sandwich annular spherical shells composed of three layers, where the top and bottom layers are made of nano-composite reinforced CNTs and a core layer is homogeneous materials. Two different models of CNT reinforcements are namely uniformly distributed (UD-UD) and functionally graded $(\mathrm{X}-\mathrm{X})$. The annular spherical shell is symmetric and the symmetry line is in mid-plane of the shell. The thickness of the homogeneous core and the face sheets are respectively presented as $h_{c}, h_{f}$, the total thickness of sandwich annular spherical shells is $h=h c+2 h_{f}$ (Fig. 1).

The volume fractions of the two distribution types, which are considered here, are expressed as follows:

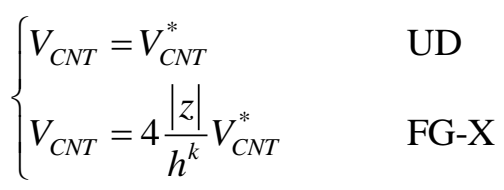

where $V_{C N T}^{*}=\frac{w_{C N T}}{w_{C N T}+\left(\rho_{C N T} / \rho_{m}\right)-\left(\rho_{C N T} / \rho_{m}\right) w_{C N T}}$, with subscript ${ }_{C N T},{ }_{m}$ are indicators which used to represent CNTs and matrix, $w$ and $\rho$ are mass fraction and density, respectively. The 
superscript $k(k=1,2,3)$ represents the top layer $-\frac{h_{c}}{2}-h_{f} \leq z \leq-\frac{h_{c}}{2}$, the core layer $-\frac{h_{c}}{2} \leq z \leq \frac{h_{c}}{2}$, and the bottom layer $\frac{h_{c}}{2} \leq z \leq \frac{h_{c}}{2}+h_{f}$.

The elastic modulus $(E)$ and shear modulus $(G)$ of the nano-composite layers can be achieved as follows [2]:

$$
\begin{aligned}
& E_{11}=E_{m} V_{m}+o_{1} E_{11}^{C N T} V_{C N T}, \\
& \frac{o_{2}}{E_{22}}=\frac{V_{m}}{E_{m}}+\frac{V_{C N T}}{E_{22}^{C N T}}, \\
& \frac{o_{3}}{G_{12}}=\frac{V_{m}}{G_{m}}+\frac{V_{C N T}}{G_{12}^{C N T}} .
\end{aligned}
$$

in which, $V$ is volume fractions. The corrective parameters $o_{i}(i=\overline{1 \div 3})$ are shown below

Table 1. The corrective parameters for CNTs-polymer composites [7]

\begin{tabular}{llll}
\hline$V_{C N T}^{*}$ & $o_{1}$ & $o_{2}$ & $o_{3}$ \\
\hline 0.12 & 0.137 & 1.022 & 0.715 \\
0.17 & 0.142 & 1.626 & 1.138 \\
0.28 & 0.141 & 1.585 & 1.110 \\
\hline
\end{tabular}

\subsection{Basic equations}

The strain fields of the shell talking von Karman's geometrical nonlinearity into account [16]:

$$
\begin{gathered}
\{\varepsilon\}=\left\{\varepsilon^{0}\right\}-z\{\chi\}, \\
\left\{\begin{array}{c}
\varepsilon_{r}^{0} \\
\varepsilon_{\theta}^{0} \\
\gamma_{r \theta}^{0}
\end{array}\right\}=\left(\begin{array}{c}
\frac{\partial u}{\partial r}-\frac{w}{R}+\frac{1}{2}\left(\frac{\partial w}{\partial r}\right)^{2} \\
\frac{1}{r} \frac{\partial v}{\partial \theta}+\frac{u}{R}-\frac{w}{R}+\frac{1}{2 r^{2}}\left(\frac{\partial w}{\partial \theta}\right)^{2} \\
\frac{\partial v}{\partial r}+\frac{1}{r} \frac{\partial u}{\partial \theta}-\frac{v}{r}+\frac{1}{r} \frac{\partial w}{\partial r} \frac{\partial w}{\partial \theta}
\end{array}\right),\left\{\begin{array}{c}
\chi_{r} \\
\chi_{\theta} \\
\chi_{r \theta}
\end{array}\right\}=\left(\begin{array}{c}
\frac{\partial^{2} w}{\partial r^{2}} \\
\frac{1}{r} \frac{\partial w}{\partial r}+\frac{1}{r^{2}} \frac{\partial^{2} w}{\partial \theta^{2}} \\
\frac{1}{r} \frac{\partial^{2} w}{\partial r \partial \theta}-\frac{1}{r^{2}} \frac{\partial w}{\partial \theta}
\end{array}\right) .
\end{gathered}
$$

The relationship between strain and stress:

$$
\{\sigma\}^{k}=[Q]^{k}\{\varepsilon\}^{k}
$$

where $Q_{\mathrm{ij}}^{k}$ are elastic coefficients. $Q_{11}^{k}=\frac{E_{11}^{k}}{1-v_{12}^{k} v_{21}^{k}}, Q_{12}^{k}=\frac{v_{21}^{k} E_{11}^{k}}{1-v_{12}^{k} v_{21}^{k}}, Q_{22}^{k}=\frac{E_{22}^{k}}{1-v_{12}^{k} v_{21}^{k}}, Q_{66}^{k}=G_{12}^{k}$.

The force and moment fields of the shell can be defined as: 


$$
\left\{\begin{array}{l}
N_{i} \\
M_{i}
\end{array}\right\}=\int_{-\frac{h_{c}}{2}-h_{f}}^{-\frac{h_{c}}{2}} \sigma_{i}^{T}\left\{\begin{array}{l}
1 \\
z
\end{array}\right\} d z+\int_{-\frac{h_{c}}{2}}^{\frac{h_{c}}{2}} \sigma_{i}^{C}\left\{\begin{array}{l}
1 \\
z
\end{array}\right\} d z+\int_{\frac{h_{c}}{2}}^{\frac{h_{c}}{2}+h_{f}} \sigma_{i}^{B}\left\{\begin{array}{l}
1 \\
z
\end{array}\right\} d z\{i=r, \theta, r \theta\} .
$$

Specifically, substituting the results of Eq. (4) to Eq. (5) leads to:

$$
\left\{\begin{array}{l}
N \\
M
\end{array}\right\}=\left(\begin{array}{ll}
{[A]} & {[B]} \\
{[B]} & {[D]}
\end{array}\right)\left\{\begin{array}{c}
\varepsilon_{0} \\
\chi
\end{array}\right\} .
$$

All the detailed coefficients are shown in Appendix.

The nonlinear equilibrium equations of the annular spherical shell are given as [16]:

$$
\begin{gathered}
\frac{\partial N_{r}}{\partial r}+\frac{1}{r} \frac{\partial N_{r \theta}}{\partial \theta}+\frac{N_{r}}{r}-\frac{N_{\theta}}{r}=0, \\
\frac{\partial N_{\theta}}{r \partial \theta}+\frac{\partial N_{r \theta}}{\partial r}+\frac{2 N_{r \theta}}{r}=0, \\
\frac{\partial^{2} M_{r}}{\partial r^{2}}+\frac{2}{r} \frac{\partial M_{r}}{\partial r}+2\left(\frac{\partial^{2} M_{r \theta}}{r \partial r \partial \theta}+\frac{1}{r^{2}} \frac{\partial M_{r \theta}}{\partial \theta}\right)+\frac{1}{r^{2}} \frac{\partial^{2} M_{\theta}}{\partial \theta^{2}}-\frac{1}{r} \frac{\partial M_{\theta}}{\partial r}+\frac{1}{R}\left(N_{r}+N_{\theta}\right)+ \\
+\frac{1}{r} \frac{\partial}{\partial r}\left(r N_{r} \frac{\partial w}{\partial r}+N_{r \theta} \frac{\partial w}{\partial \theta}\right)+\frac{1}{r} \frac{\partial}{\partial \theta}\left(N_{r \theta} \frac{\partial w}{\partial r}+\frac{N_{\theta}}{r} \frac{\partial w}{\partial \theta}\right)+q-q_{E}=0 .
\end{gathered}
$$

where $q_{E}=k_{1} w-k_{2} \Delta w, \Delta w=\frac{\partial^{2} w}{\partial r^{2}}+\frac{1}{r} \frac{\partial w}{\partial r}+\frac{1}{r^{2}} \frac{\partial^{2} w}{\partial \theta^{2}} ; \quad w$ is the deflection of the shell, $k_{1}\left(N / \mathrm{m}^{3}\right)$ isthe spring layer foundation stiffness and $k_{2}(N / m)$ is the shear layer foundation stiffness of Pasternak foundation.

The stress function method is applied in this paper, so the selected stress function must be satisfied: $N_{r}=\frac{1}{r} \frac{\partial F}{\partial r}+\frac{1}{r^{2}} \frac{\partial^{2} F}{\partial \theta^{2}}, N_{\theta}=\frac{\partial^{2} F}{\partial r^{2}}, N_{r \theta}=-\frac{1}{r} \frac{\partial^{2} F}{\partial r \partial \theta}+\frac{1}{r^{2}} \frac{\partial F}{\partial \theta}$.

A transformation has been used for the FGM annular spherical shell in [16], reused in this study to simplify the calculation process such as :

$$
w=w(\varsigma), F=F_{0}(\varsigma) \mathrm{e}^{2 \varsigma} \text { with } r=r_{0} \mathrm{e}^{\varsigma} ; \varsigma=\ln \frac{r}{r_{0}} ; \frac{\partial r}{\partial \varsigma}=r_{0} e^{\varsigma} ; \frac{\partial \varsigma}{\partial r}=\frac{1}{r}
$$

The annular spherical shell's compatibility equation is shown:

$$
\frac{1}{r^{2}} \frac{\partial^{2} \varepsilon_{r}^{0}}{\partial \theta^{2}}-\frac{1}{r} \frac{\partial \varepsilon_{r}^{0}}{\partial r}+\frac{1}{r^{2}} \frac{\partial}{\partial r}\left(r^{2} \frac{\partial \varepsilon_{\theta}^{0}}{\partial r}\right)-\frac{1}{r^{2}} \frac{\partial^{2}}{\partial r \partial \theta}\left(r \gamma_{r \theta}^{0}\right)=-\frac{\Delta w}{r}+\chi_{r \theta}^{2}-\chi_{r} \chi_{\theta},
$$

Substituting the results of Eq. (6) into Eqs. (7) and (8), after a complex series of calculations yields:

$$
\begin{gathered}
T_{1}^{*}+T_{2}^{*}+T_{3}^{*}=0 \\
T_{4}^{*}+T_{5}^{*}+T_{6}^{*}+q-q_{E}=\rho_{1} \frac{\partial^{2} w}{\partial t^{2}}
\end{gathered}
$$


where the $T_{i}^{*}(i=1-6)$ coefficients are shown as:

$$
\begin{aligned}
& T_{1}^{*}=\frac{1}{r_{0}^{4} e^{2 \varsigma}}\left\{\begin{array}{l}
A_{11}^{*} \frac{\partial^{4} F_{0}}{\partial \theta^{4}}+\left(2 A_{11}^{*}+2 A_{12}^{*}+A_{66}^{*}\right) \frac{\partial^{2} F_{0}}{\partial \theta^{2}}+2 \frac{\partial F_{0}}{\partial \varsigma}\left(A_{22}^{*}-2 A_{11}^{*}\right)+4 A_{22}^{*} \frac{\partial^{3} F_{0}}{\partial \varsigma^{3}} \\
+\frac{\partial^{2} F_{0}}{\partial \varsigma^{2}}\left(5 A_{22}^{*}-A_{11}^{*}\right)+A_{22}^{*} \frac{\partial^{4} F_{0}}{\partial \varsigma^{4}}+\frac{\partial^{4} F_{0}}{\partial \varsigma^{2} \partial \theta^{2}}\left(2 A_{12}^{*}+A_{66}^{*}\right)+\frac{2 \partial^{3} F_{0}}{\partial \varsigma \partial \theta^{2}}\left(2 A_{12}^{*}+A_{66}^{*}\right)
\end{array}\right\}, \\
& T_{2}^{*}=\frac{1}{r_{0}^{4} e^{4 \varsigma}}\left\{\begin{array}{l}
B_{12}^{*} \frac{\partial^{4} w}{\partial \theta^{4}}+\left(2 B_{12}^{*}+2 B_{22}^{*}-B_{66}^{*}+r_{0} e^{\varsigma}\right) \frac{\partial^{2} w}{\partial \theta^{2}}+\frac{\partial w}{\partial \varsigma}\left[-2 B_{21}^{*}+2\left(B_{22}^{*}-B_{11}^{*}\right)+2 B_{12}^{*}\right. \\
+\frac{\partial^{2} w}{\partial \varsigma^{2}}\left[5 B_{21}^{*}-3\left(B_{22}^{*}-B_{11}^{*}\right)-\left(B_{12}^{*}-r_{0} e^{\varsigma}\right)\right]+\frac{\partial^{3} w}{\partial \varsigma^{3}}\left[-4 B_{21}^{*}+B_{22}^{*}-B_{11}^{*}\right]+B_{21}^{*} \frac{\partial^{4} w}{\partial \varsigma^{4}} \\
+\left(B_{11}^{*}+B_{22}^{*}-B_{66}^{*}\right) \frac{\partial^{4} w}{\partial \varsigma^{2} \partial \theta^{2}}+\frac{\partial^{3} w}{\partial \varsigma \partial \theta^{2}}\left[-B_{11}^{*}+2 B_{66}^{*}-3 B_{22}^{*}\right]
\end{array}\right\}, \\
& T_{3}^{*}=\frac{1}{r_{0}^{4} e^{4 \varsigma}}\left\{-\left(\frac{\partial^{2} w}{\partial \varsigma \partial \theta}-\frac{\partial w}{\partial \theta}\right)^{2}+\left(-\frac{\partial w}{\partial \varsigma}+\frac{\partial^{2} w}{\partial \varsigma^{2}}\right)\left(\frac{\partial w}{\partial \varsigma}+\frac{\partial^{2} w}{\partial \theta^{2}}\right)\right\}, \\
& T_{4}^{*}=\frac{1}{r_{0}^{4} e^{2 \varsigma}}\left\{\begin{array}{l}
C_{21} \frac{\partial^{4} F_{0}}{\partial \theta^{4}}+2\left(-C_{31}+C_{21}+C_{22}\right) \frac{\partial^{2} F_{0}}{\partial \theta^{2}}+2\left(C_{11}+C_{12}-C_{22}-C_{21}\right) \frac{\partial F_{0}}{\partial \varsigma}+ \\
+\left(C_{11}+4 C_{12}-C_{22}\right) \frac{\partial^{3} F_{0}}{\partial \varsigma^{3}}+\left(5 C_{12}+3\left(C_{11}-C_{22}\right)-C_{21}\right) \frac{\partial^{2} F_{0}}{\partial \varsigma^{2}}+C_{12} \frac{\partial^{4} F_{0}}{\partial \varsigma^{4}}+ \\
+\left(C_{11}-4 C_{31}+3 C_{22}\right) \frac{\partial^{3} F_{0}}{\partial \varsigma \partial \theta^{2}}+\left(C_{11}-2 C_{31}+C_{22}\right) \frac{\partial^{4} F_{0}}{\partial \varsigma^{2} \partial \theta^{2}}
\end{array}\right\}, \\
& T_{5}^{*}=\frac{1}{r_{0}^{4} e^{4 \varsigma}}\left\{\begin{array}{l}
\mathrm{C}_{24} \frac{\partial^{4} w}{\partial \theta^{4}}+2\left(C_{14}+C_{23}+C_{24}\right) \frac{\partial^{2} w}{\partial \theta^{2}}+2\left(C_{14}-C_{23}-C_{13}+C_{24}\right) \frac{\partial w}{\partial \varsigma}+ \\
+\left(5 C_{13}-3\left(C_{14}-C_{23}\right)-C_{24}\right) \frac{\partial^{2} w}{\partial \varsigma^{2}}+\left(-4 C_{13}+C_{14}-C_{23}\right) \frac{\partial^{3} w}{\partial \varsigma^{3}}+C_{13} \frac{\partial^{4} w}{\partial \varsigma^{4}}+ \\
+\left(-3 C_{14}-4 C_{32}-C_{23}\right) \frac{\partial^{3} w}{\partial \varsigma \partial \theta^{2}}+\left(C_{14}+2 C_{32}+C_{23}\right) \frac{\partial^{4} w}{\partial \varsigma^{2} \partial \theta^{2}}
\end{array}\right\}, \\
& T_{6}^{*}=\frac{1}{r_{0}^{4} e^{2 \varsigma}}\left[\begin{array}{l}
\left(\frac{\partial^{2} F_{0}}{\partial \varsigma^{2}}+2 \frac{\partial F_{0}}{\partial \varsigma}-\frac{\partial^{2} F_{0}}{\partial \theta^{2}}\right) \frac{\partial w}{\partial \varsigma}+\left(\frac{\partial F_{0}}{\partial \varsigma}+2 F_{0}+\frac{\partial^{2} F_{0}}{\partial \theta^{2}}\right) \frac{\partial^{2} w}{\partial \varsigma^{2}}+2\left(\frac{\partial^{2} F_{0}}{\partial \varsigma \partial \theta}+\frac{\partial F_{0}}{\partial \theta}\right) \frac{\partial w}{\partial \theta} \\
-2\left(\frac{\partial^{2} F_{0}}{\partial \varsigma \partial \theta}+\frac{\partial F_{0}}{\partial \theta}\right) \frac{\partial^{2} w}{\partial \varsigma \partial \theta}+\left(\frac{\partial^{2} F_{0}}{\partial \varsigma^{2}}+3 \frac{\partial F_{0}}{\partial \varsigma}+2 F_{0}\right) \frac{\partial^{2} w}{\partial \theta^{2}}
\end{array}\right]+ \\
& +\frac{1}{R} \frac{1}{r_{0}^{2}}\left(4 \frac{\partial F_{0}}{\partial \varsigma}+4 F_{0}+\frac{\partial^{2} F_{0}}{\partial \theta^{2}}+\frac{\partial^{2} F_{0}}{\partial \varsigma^{2}}\right) \text {, } \\
& \Delta w=\frac{\partial^{2} w}{\partial r^{2}}+\frac{1}{r} \frac{\partial w}{\partial r}+\frac{1}{r^{2}} \frac{\partial^{2} w}{\partial \theta^{2}}=\frac{1}{r^{2}} \frac{\partial^{2} w}{\partial \varsigma^{2}}+\frac{1}{r^{2}} \frac{\partial^{2} w}{\partial \theta^{2}}=\frac{1}{r_{0}^{2} e^{2 \varsigma}}\left(\frac{\partial^{2} w}{\partial \varsigma^{2}}+\frac{\partial^{2} w}{\partial \theta^{2}}\right) .
\end{aligned}
$$


The Eqs. (9a, 9b) with the new expressions $T_{i}^{*},(i=1, \ldots, 6)$ are basic equations to analyze the dynamic response of annular spherical shells resting on elastic foundations.

\subsection{Analytical solution}

The shell is assumed to be simply supported along the periphery and subjected to mechanical loads uniformly distributed on the outer surface and the base edges of the shell. Depending on the in-plane behavior at the edge of boundary conditions will be considered in case the edges are simply supported and immovable. For this case, the boundary conditions are

$$
u=0, w=0, \frac{\partial^{2} w}{\partial \varsigma^{2}}-\frac{\partial w}{\partial \varsigma}=0, N_{r}=0, N_{r \theta}=0, \text { with } \varsigma=0 \text { (i.e at } r=r_{0} \text { ). }
$$

with $N_{0}$ is the fictitious compressive load rendering the immovable edges

The solution of the annual spherical shell satisfied the boundary condition can be chosen as:

$$
w=W e^{\varsigma} \sin \left(\beta_{1} \varsigma\right) \sin (n \theta),
$$

in which $\beta_{1}=m \pi / a, a=\ln r_{1} / r_{0}$, and $m, n$ are buckling mode parameters in the $r$ and $\theta$ directions, respectively.

By using the supper position method, the form of stress function $F_{0}$ for the nano-composite annular spherical shells is given as:

$$
\begin{aligned}
& F_{0}=f_{1} \sin \left(2 \beta_{1} \varsigma\right)+\left(f_{21}+\mathrm{e}^{-\varsigma} f_{22}\right) \sin (n \theta) \sin \left(\beta_{1} \varsigma\right)+f_{3} \cos (2 n \theta) \sin \left(2 \beta_{1} \varsigma\right)+f_{4} \cos (2 n \theta) \\
& +f_{5} \cos \left(2 \beta_{1} \varsigma\right)+\left(f_{61}+f_{62} \mathrm{e}^{-\varsigma}\right) \sin (n \theta) \cos \left(\beta_{1} \varsigma\right)+f_{7} \cos (2 n \theta) \cos \left(2 \beta_{1} \varsigma\right),
\end{aligned}
$$

in which, $f_{i}(i=1-7)$ are obtained by solving the geometrical compatibility equation talking stress function and approximate solution into account.All the detailed coefficients are shown in Appendix.

Applying Galerkin method for the nano-composite sandwich annular spherical shell:

$$
\int_{0}^{\ln \frac{r_{1}}{r_{0}}} \int_{0}^{2 \pi} \Phi e^{\varsigma} \sin \left(\beta_{1} \varsigma\right) \sin (n \theta) d \varsigma d \theta=0
$$

where $\Phi$ is the Eq. (9b) after introducing Eq.(12) and Eq.(11).

The differential equation is obtained to analysis the nonlinear dynamic response as follow:

$$
\frac{d^{2} W}{d t^{2}}+\left(\frac{8 A_{1}}{r_{0}^{4} B_{4}}\right) W^{3}+\frac{8\left(A_{3} R+A_{2} r_{0}^{2}\right)}{R_{0}{ }^{4} B_{4}} W^{2}+8\left[\frac{A_{5}}{R r_{0}^{2} B_{4}}+\frac{A_{6}}{r_{0}^{4} B_{4}}-\frac{\left(B_{1} k_{1}+B_{2} k_{2}\right)}{B_{4}}\right] W=\left(\frac{8 B_{3}}{B_{4}}\right) q
$$

\section{Numerical results and discussion}

The sandwich-structured composite shells composed of three layers. The core layer is poly (methyl methacrylate)(PMMA) with material properties shown as [7]: $E_{m}(G P a)=(3.52-0.0034 \Delta \mathrm{T}), v_{m}=0.34$. Two face layers are made of composite reinforced CNTs with PMMA is considered as the matrix. The material properties of SWCNTs are shown as [17]: 


$$
\begin{aligned}
& E_{11}^{C N T}(T P a)=6.3998-4.338417 \times 10^{-3} T+7.43 \times 10^{-6} T^{2}-4.458333 \times 10^{-9} T^{3}, \\
& E_{22}^{C N T}(T P a)=8.02155-5.420375 \times 10^{-3} T+9.275 \times 10^{-6} T^{2}-5.5625 \times 10^{-9} T^{3}, \\
& G_{12}^{C N T}(T P a)=1.40755+3.476208 \times 10^{-3} T-6.965 \times 10^{-6} T^{2}+4.479167 \times 10^{-9} T^{3}, \\
& v_{12}^{C N T}=0.175, \rho^{C N T}=1400 \mathrm{Kg} / \mathrm{m}^{3} .
\end{aligned}
$$

\subsection{Natural frequencies}

Table 2. The effect of $R / h$ ration and $V_{C N T}^{*}$ on the natural frequencies $\omega(1 / s)$ of nano-composite sandwich annular spherical shells

\begin{tabular}{cllll}
\hline \multirow{2}{*}{$\frac{V_{C N T}^{*}=0.12}{3}$} & \multicolumn{3}{c}{$V_{C N T}^{*}=0.28$} \\
\hline 200 & 1034.10 & $U D-U D$ & $X-X$ & $U D-U D$ \\
250 & 827.34 & 505.95 & 1284.80 & 724.16 \\
300 & 691.48 & 410.65 & 1025.90 & 582.37 \\
500 & 425.84 & 349.04 & 855.51 & 489.61 \\
\hline
\end{tabular}

From the table, it's clear that the natural frequencies are significantly changed with different types of $R / h$ ratio, $V_{C N T}^{*}$ and CNT reinforcements. The table indicates that the natural frequencies increases when the ratio $R / h$ decreases and $V_{C N T}^{*}$ increases. In addition, the natural frequencies of the $\mathrm{X}-\mathrm{X}$ model have higher than the UD-UD mode.

\subsection{Dynamic response analysis}

The reaction of the sandwich-structured shell with two models of CNT reinforcements (UD-UD, $\mathrm{X}-\mathrm{X}$ ) is shown in figure 2. In this figure, the frequencies of the loading are approximately similar to the natural frequencies of the nano-composite sandwich annular spherical shell (shown in table 2). The frequency of loading is $\omega_{\text {force }}=750(1 / s)$ and $\omega_{\text {force }}=350(1 / s)$ for the X-X and UD-UD models, respectively.

The effect of the core to face sheet thickness ratio $h_{c} / h_{f}$ on the deflection-time curveof the nanocomposite sandwich shell is considered in both X-X and UD-UD models in figure 3. For the X-X type, when the $h_{c} / h_{f}$ ratio is increased, the amplitude of vibration decreases. On the contrary, for the UDUD model, the amplitude of fluctuation increases as the ratio increases.

The influence of CNTs' volume fraction $V_{C N T}^{*}$ on the dynamic response of nano-composite sandwich annular spherical shell in figure 4. It shows that the fluctuates of the nano-composite sandwich annular spherical shell increase when the volume fraction $V_{C N T}^{*}$ decrease. In addition, it is possible to see the deflection amplitude of the X-X model is smaller than the UD-UD model. In other words, the X-X model has better load-bearing capacity than UD-UD model.

Figure 5 presents the influence of $R / h$ ratio on the deflection-time curve of the nano-composite sandwich annular spherical shell reinforced by CNTs. It's clear that the strength of the sandwichstructure shell is better with lower $R / h$ ratio. 


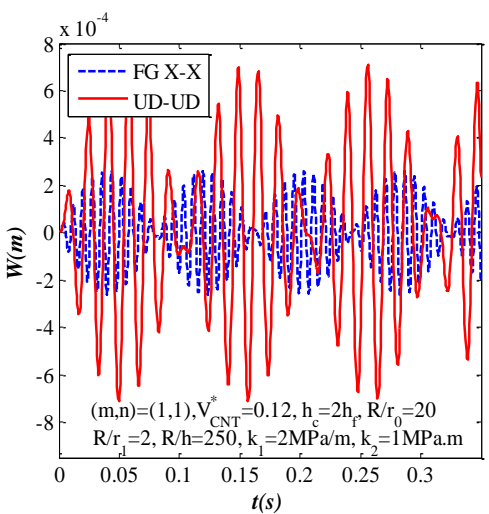

Fig.2. Effect of CNT models on the nonlinear dynamical response.

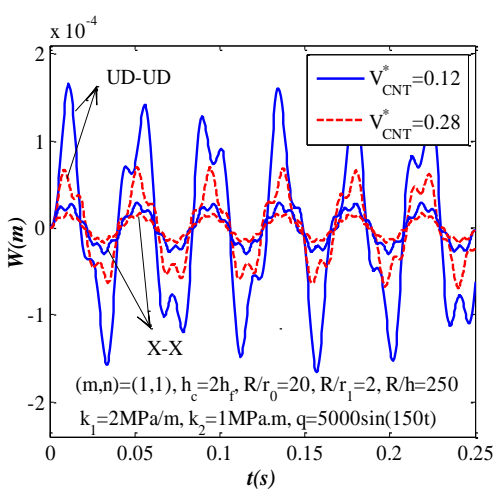

Fig. 4. Effect of CNTs volume fraction on the deflection-time curve.

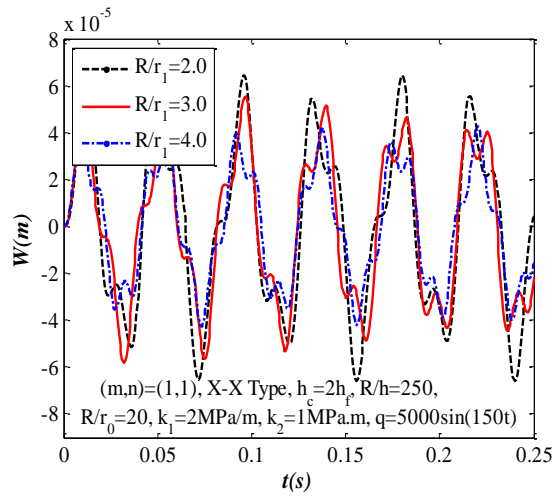

Fig. 6. Effect of $R / r_{1}$ ratio on the deflection-time curve.

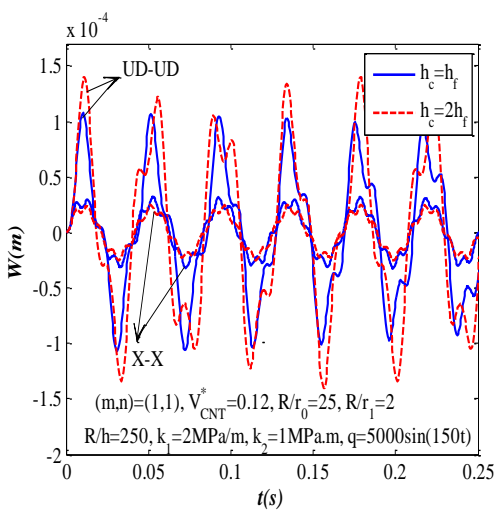

Fig. 3. Effect of core to face sheet thicknessratio $h_{c} / h_{f}$ on the nonlinear dynamical response.

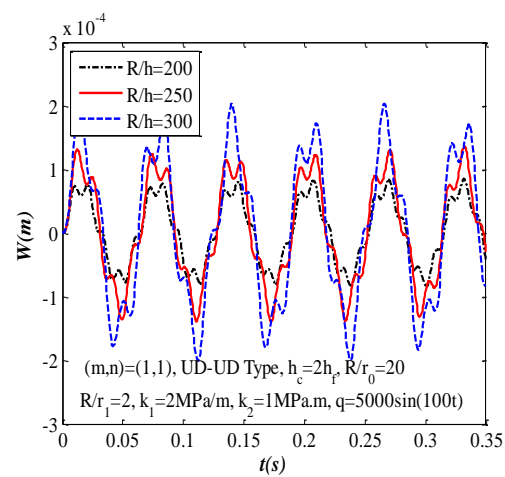

Fig. 5. Effect of geometric ratio $R / h$ on the deflection-time curve.

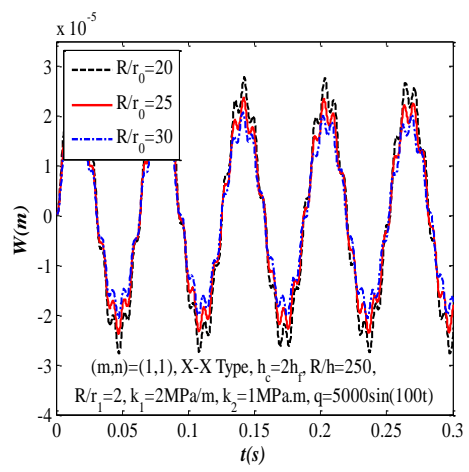

Fig. 7. Effect of $R / r_{0}$ ratio on the deflection-time curve.

Figures 6 and 7 indicate the influence of curvature to radii ratios $\left(R / r_{1}, R / r_{0}\right)$ on the dynamic response of the nano-composite sandwich annular spherical shell. Clearly, the fluctuation of the shell is affected by the curvature to radius ratio. 


\section{Conclusions}

In this study, by using the supper position method, the form of stress function has found to solve the problem of the nanocomposite annular spherical shells. The conclusions are obtained:

The deflection-time curve and natural frequencies of the nano-composite sandwich shells are considerably changed by geometrical parameters.

Among the CNT reinforcement models, the X-X model is the best for strength of the sandwich annular spherical shell.

CNTs' volume fraction does wonder for the load capacity of the annular spherical shell.

\section{Acknowledgments}

This research is funded by Vietnam National Foundation for Science and Technology Development (NAFOSTED) under grant number 107.02-2018.314. The authors are grateful for this support.

\section{References}

[1] S. Iijima, Helical microtubules of graphitic carbon, Nature 354 (6348) (1991) 56.

[2] H.S. Shen, Nonlinear bending of functionally graded carbon nanotube-reinforced composite plates in thermal environments,Compos. Struct., 91 (1) (2009) 9-19.

[3] A. Farzam, B. Hassani, Thermal and mechanical buckling analysis of FG carbon nanotube reinforced composite plates using modified couple stress theory and isogeometric approach,Compos. Struct. 206 (2018) 774-790.

[4] L.T.N. Trang, H. Tung, Buckling and postbuckling of carbon nanotube-reinforced composite cylindrical panels subjected to axial compression in thermal environments, Vietnam Journal of Mechanics 40(1) (2018) 47-61.

[5] R. Zhong, Q. Wang, J. Tang, C. Shuai, B. Qin, Vibration analysis of functionally graded carbon nanotube reinforced composites (FG-CNTRC) circular, annular and sector plates,Compos. Struct. 194 (2018) 49-67.

[6] A. Frikha, S. Zghal, F. Dammak, Dynamic analysis of functionally graded carbon nanotubes-reinforced plate and shell structures using a double directors finite shell element, Aerosp. Sci. Technol. 78 (2018) 438-451.

[7] N.V. Thanh, N.D. Khoa, N.D. Tuan, P. Tran, N.D. Duc, Nonlinear dynamic response and vibration of functionally graded carbon nanotube-reinforced composite (FG-CNTRC) shear deformable plates with temperature-dependent material properties and surrounded on elastic foundations,J. Therm. Stress. 40 (10) (2017) 1254-1274.

[8] R. Moradi-Dastjerdi, F. Aghadavoudi, Static analysis of functionally graded nano-composite sandwich plates reinforced by defected CNT,Compos. Struct. 200 (2018) 839-848.

[9] Z.X. Wang, H.S. Shen,Nonlinear vibration and bending of sandwich plates with nanotube-reinforced composite face sheets, Compos Part B-Eng. 43 (2012) 411- 421.

[10] H.S. Shen, H. Wang, D.Q. Yang, Vibration of thermally postbuckled sandwich plates with nanotube-reinforced composite face sheets resting on elastic foundations, Int. J. Mech. SCI. 124-125 (2018) 253- 262.

[11] S.K. Jalali, M Heshmati,Buckling analysis of circular sandwich plates with tapered cores and functionally graded carbon nanotubes-reinforced composite face sheets, Thin-walled struct. 100 (2016)14-24.

[12] R.S. Alwar, M.C. Narasimhan,Axisymmetric non-linear analysis of laminated orthotropic annular spherical shells, Int. J. Nonlinear. Mech. 27 (1992) 611-622

[13] Dumir PC, Dube GP, Mallick A. Axisymmetric buckling of laminated thick annular spherical cap. Commun. Nonlinear. Sci .Numer. Simul. 10 (2015) 191-204.

[14] H. Li, F. Pang, H. Chen, A semi-analytical approach to analyze vibration characteristics of uniform and stepped annular-spherical shells with general boundary conditions, Eur. J. Mech. A Solids 74 (2019) 48-65. 
[15] N.D. Duc, D.H. Bich, V.T. Thuy Anh. On the nonlinear stability of eccentrically stiffened functionally graded annular spherical segment shells. Thin-Walled Struct. 106 (2016) 258-267.

[16] V.T. Thuy Anh, D.H. Bich, N.D. Duc. Nonlinear stability analysis of thin FGM annular spherical shells on elastic foundations under external pressure and thermal loads. Eur. J. Mech. A Solids. 50 (2015) 28-38.

[17] M. Mirzaei, Y Kiani. Thermal buckling of temperature dependent FG-CNT reinforced composite conical shells. Aerosp. Sci. Tech. 47 (2015) 42-53.

\section{Appendix}

$$
\begin{aligned}
& f_{1}=\frac{1}{16} \frac{a_{11} W^{2}}{a_{12}} ; f_{3}=-\frac{W^{2}\left(a_{42} a_{43}+a_{41} a_{44}\right)}{a_{42}{ }^{2}+a_{41}{ }^{2}} ; f_{4}=-\frac{a_{62} W^{2}}{a_{61}} ; f_{5}=-\frac{1}{16} \frac{W^{2} a_{51}}{a_{12}} ; f_{62}=-\frac{a_{25} W}{a_{23}} ; \\
& f_{61}=\frac{W\left(-a_{22} a_{24}+a_{21} a_{84}\right)}{a_{22}{ }^{2}+a_{21}{ }^{2}} ; f_{7}=-\frac{W^{2}\left(a_{41} a_{43}-a_{44} a_{42}\right)}{a_{42}{ }^{2}+a_{41}{ }^{2}} ; f_{21}=-\frac{W\left(a_{21} a_{24}+a_{84} a_{22}\right)}{a_{22}{ }^{2}+a_{21}{ }^{2}} ; f_{22}=-\frac{a_{85} W}{a_{23}} ; \\
& a_{11}=\left(12 A_{11} \beta_{1}^{2} n^{2}-4 A_{11} \beta_{1}^{4}+3 A_{11} n^{2}+A_{22} n^{2}-7 A_{11} \beta_{1}^{2}-A_{22} \beta_{1}^{2}-3 A_{11}-A_{22}\right) \beta_{1} \text {; } \\
& a_{12}=\left(\begin{array}{l}
16 \beta_{1}^{6} A_{11}{ }^{2}+24 A_{11}{ }^{2} \beta_{1}^{4}+8 \beta_{1}^{4} A_{22} A_{11}+9 A_{11}{ }^{2} \beta_{1}^{2}+6 A_{22} A_{11} \beta_{1}^{2}+\beta_{1}^{2} A_{22}{ }^{2} \\
+A_{11}{ }^{2}-2 A_{22} A_{11}+A_{22}{ }^{2}
\end{array}\right) ; \\
& a_{21}=\left(-2 n^{2} A_{12} \beta_{1}-n^{2} A_{66} \beta_{1}+2 A_{11} \beta_{1}^{3}-\beta_{1} A_{11}+\beta_{1} A_{22}\right) \text {; } \\
& a_{22}=\left(\begin{array}{l}
n^{2} A_{12} \beta_{1}^{2}+5 / 2 A_{11} \beta_{1}^{2}-1 / 2 A_{22} \beta_{1}^{2}+A_{22} n^{2}-n^{2} A_{12}-1 / 2 n^{2} A_{66} \\
-1 / 2 A_{22} n^{4}-1 / 2 A_{11} \beta_{1}^{4}+1 / 2 n^{2} A_{66} \beta_{1}^{2}
\end{array}\right) ; \\
& a_{23}=A_{22} n^{2}+n^{2} A_{12} \beta_{1}^{2}-0.5\left(A_{22} \beta_{1}^{2}+A_{11} \beta_{1}^{2}+A_{22}-n^{2} A_{66} \beta_{1}^{2}+A_{11} \beta_{1}^{4}+A_{22} n^{4}\right) ; a_{24}=\beta_{1} r_{0}, \\
& a_{25}=\beta_{1} B_{21}-1 / 2\left(\beta_{1} B_{11}+\beta_{1}^{3} B_{22}-n^{2} \beta_{1} B_{22}+\beta_{1} B_{22}+n^{2} \beta_{1} B_{11}-\beta_{1}^{3} B_{11}\right) ; a_{84}=1 / 2\left(r_{0}-n^{2} r_{0}-\beta_{1}^{2} r_{0}\right) \text {; }
\end{aligned}
$$

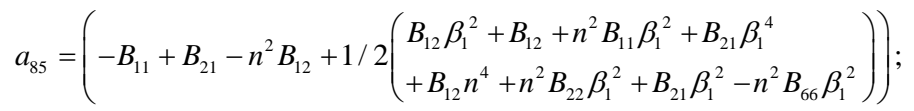

$$
\begin{aligned}
& a_{41}=\left(-16 n^{2} \beta_{1} A_{12}-8 n^{2} \beta_{1} A_{66}+16 A_{11} \beta_{1}^{3}-2 \beta_{1} A_{11}+2 \beta_{1} A_{22}\right) \\
& a_{42}=\left(\begin{array}{l}
8 A_{22} n^{4}-16 n^{2} A_{12} \beta_{1}^{2}-8 n^{2} A_{66} \beta_{1}^{2}+8 A_{11} \beta_{1}^{4}+4 n^{2} A_{12}-4 A_{22} n^{2} \\
+2 n^{2} A_{66}-10 A_{11} \beta_{1}^{2}+2 A_{22} \beta_{1}^{2}
\end{array}\right) \\
& a_{43}=\left(-1 / 8 \beta_{1}^{3}-1 / 8 n^{2} \beta_{1}+1 / 8 \beta_{1}\right), a_{44}=\left(-1 / 4 \beta_{1}^{2}\right) \\
& a_{51}=\left(\begin{array}{l}
8 \beta_{1}^{4} A_{11} n^{2}-2 A_{11} \beta_{1}^{2} n^{2}+2 \beta_{1}^{2} A_{22} n^{2}-A_{11} n^{2}+A_{22} n^{2}+A_{11} \beta_{1}^{2} \\
-A_{22} \beta_{1}^{2}+A_{11}-A_{22}
\end{array}\right) \\
& a_{61}=\left(-16 A_{22} n^{4}-8 n^{2} A_{12}+8 A_{22} n^{2}-4 n^{2} A_{66}\right), a_{62}=\left(-1 / 2 n^{2} \beta_{1}^{2}\right) \\
& A_{1}=\frac{\pi a_{62}\left(2 \pi^{2} n^{2} m^{2}-m^{2} \pi^{2}-n^{2} a^{2}+a^{2}\right)}{4 a_{61} a} \\
& +\frac{\pi a_{51}\left(2 \pi^{2} n^{2} m^{2}+m^{2} \pi^{2}-n^{2} a^{2}+a^{2}\right)}{64 a_{12} a}-\frac{\pi^{2} m a_{11}\left(m^{2} \pi^{2}-3 n^{2} a^{2}+a^{2}\right)}{64 a_{12} a^{2}} \\
& -\frac{\pi\left(m^{2} \pi^{2}+a^{2}-n^{2} a^{2}\right)\left(a_{41} a_{43}-a_{44} a_{42}\right)}{8\left({a_{41}}^{2}+{a_{42}}^{2}\right) a}-\frac{\pi^{2} m\left(m^{2} \pi^{2}+a^{2}+n^{2} a^{2}\right)\left(a_{41} a_{44}+a_{42} a_{43}\right)}{8\left({a_{41}}^{2}+{a_{42}}^{2}\right) a^{2}} ;
\end{aligned}
$$




$$
\begin{aligned}
& A_{2}=\frac{-8 \pi a m\left(n^{2}-1\right)\left(1+\mathrm{e}^{a}\right) a_{62}}{3\left(m^{2} \pi^{2}+a^{2}\right) n a_{61}}-\frac{\pi m a_{51}\left(3 m^{2} \pi^{2}+a^{2}\right)\left(1+\mathrm{e}^{a}\right)}{2 a n a_{12}\left(9 m^{2} \pi^{2}+a^{2}\right)}-\frac{\pi^{2} m^{2}\left(1+\mathrm{e}^{a}\right) a_{11}}{\left(9 m^{2} \pi^{2}+a^{2}\right) n a_{12}}+ \\
& -\frac{16 \pi^{2} m^{2}\left(m^{2} \pi^{2}-2 n^{2} a^{2}+a^{2}\right)\left(1+\mathrm{e}^{a}\right)\left(a_{41} a_{44}+a_{42} a_{43}\right)}{3\left(m^{2} \pi^{2}+a^{2}\right)\left(9 m^{2} \pi^{2}+a^{2}\right) n\left(a_{41}{ }^{2}+a_{42}{ }^{2}\right)}+ \\
& +\frac{8 \pi m\left(3 m^{4} \pi^{4}+3 a^{2} m^{2} \pi^{2} n^{2}+4 a^{2} m^{2} \pi^{2}-n^{2} a^{4}+a^{4}\right)\left(1+\mathrm{e}^{a}\right)\left(a_{41} a_{43}-a_{44} a_{42}\right)}{3 a\left(m^{2} \pi^{2}+a^{2}\right)\left(9 m^{2} \pi^{2}+a^{2}\right) n\left(a_{41}^{2}+a_{42}^{2}\right)} ; \\
& A_{3}=\frac{\pi^{3} m^{3} a_{51}\left(\mathrm{e}^{-a}+1\right)\left[\begin{array}{l}
12 \pi^{4} m^{4} C_{12}+a^{4}\left(7 C_{11}+9 C_{12}-5 C_{21}-7 C_{22}\right) \\
+\pi^{2} a^{2} m^{2}\left(17 C_{22}-17 C_{11}-51 C_{12}+3 C_{21}\right)+
\end{array}\right]}{2 n a^{3}\left(m^{2} \pi^{2}+a^{2}\right)\left(9 m^{2} \pi^{2}+a^{2}\right) a_{12}}+ \\
& -\frac{4 \pi^{2} m^{2}\left(\mathrm{e}^{-a}+1\right)\left[\begin{array}{l}
\pi^{3} a m^{3} a_{85}\left(9 n^{2}-10\right)+\pi a^{3} m a_{85}\left(-5 n^{2}+6\right) \\
+3 \pi^{2} a^{2} m^{2} a_{25}\left(3 n^{2}-4\right)+a^{4} a_{25}\left(1-n^{2}\right)+3 \pi^{4} m^{4} a_{25}
\end{array}\right]}{3 a_{23} a^{2}\left(\pi^{2} m^{2}+a^{2}\right)\left(9 \pi^{2} m^{2}+a^{2}\right) n}+ \\
& +\frac{8\left[\begin{array}{l}
a\left(-3 \pi^{2} m^{2} n^{2}+2 \pi^{2} m^{2}+4 a^{2} n^{2}\right)\left(a_{21} a_{24}+a_{22} a_{84}\right)+ \\
\left(\pi a^{2} m\left(2 n^{2}-1\right)-4 a^{3}+\pi^{3} m^{3}\right)\left(a_{21} a_{84}-a_{22} a_{24}\right)
\end{array}\right]}{9 m\left(a_{21}{ }^{2}+a_{22}{ }^{2}\right) a^{2} n \pi}+ \\
& -\frac{16 \pi a m n\left(\mathrm{e}^{-a}+1\right)\left(2 n^{2} C_{21}-C_{21}-C_{22}+C_{31}\right)}{3\left(\pi^{2} m^{2}+a^{2}\right)}+ \\
& +\frac{m^{2} \pi^{2}\left(\mathrm{e}^{-a}+1\right) a_{11}}{2 a^{2}\left(m^{2} \pi^{2}+a^{2}\right)\left(9 m^{2} \pi^{2}+a^{2}\right) n a_{12}}\left(\begin{array}{l}
\pi^{4} m^{4}\left(6 C_{11}+40 C_{12}-6 C_{22}\right) \\
+\pi^{2} a^{2} m^{2}\left(-17 C_{11}-31 C_{12}+7 C_{21}+17 C_{22}\right) \\
+a^{4}\left(C_{11}+C_{12}-C_{21}-C_{22}\right)
\end{array}\right)+ \\
& -\frac{8 \pi m\left(\mathrm{e}^{-a}+1\right)\left(a_{41} a_{43}-a_{44} a_{42}\right)}{3 n a^{3}\left(\pi^{2} m^{2}+a^{2}\right)\left(9 \pi^{2} m^{2}+a^{2}\right)\left(a_{41}^{2}+a_{42}^{2}\right)}\left(\begin{array}{l}
12 \pi^{4} a^{2} m^{4} n^{2}\left(C_{11}+C_{22}-2 C_{31}\right) \\
+\pi^{4} a^{2} m^{4}\left(3 C_{21}+17 C_{22}-17 C_{11}-51 C_{12}\right) \\
+12 \pi^{6} m^{6} C_{12}+12 \pi^{2} a^{4} m^{2} n^{4} C_{21}-4 a^{6} n^{4} C_{21} \\
+\pi^{2} a^{4} m^{2} n^{2}\left(46 C_{31}-12 C_{11}-6 C_{21}-34 C_{22}\right) \\
+\pi^{2} a^{4} m^{2}\left(7 C_{11}+9 C_{12}-5 C_{21}-7 C_{22}\right) \\
+2 a^{6} n^{2}\left(C_{21}+C_{22}-C_{31}\right)
\end{array}\right) \\
& +\frac{8 \pi^{2} m^{2}\left(\mathrm{e}^{-a}+1\right)\left(a_{41} a_{44}+a_{42} a_{43}\right)}{3 n a^{2}\left(\pi^{2} m^{2}+a^{2}\right)\left(9 \pi^{2} m^{2}+a^{2}\right)\left(a_{41}^{2}+a_{42}^{2}\right)}\left[\begin{array}{l}
\pi^{4} m^{4}\left(6 C_{11}+40 C_{12}-6 C_{22}\right)+16 a^{4} n^{4} C_{21} \\
+\pi^{2} a^{2} m^{2} n^{2}\left(22 C_{11}+34 C_{22}-56 C_{31}\right) \\
-\pi^{2} a^{2} m^{2}\left(17 C_{11}+31 C_{12}-7 C_{21}-17 C_{22}\right) \\
-a^{4} n^{2}\left(2 C_{11}+8 C_{21}+14 C_{22}-16 C_{31}\right) \\
+a^{4}\left(C_{11}+C_{12}-C_{21}-C_{22}\right)
\end{array}\right]
\end{aligned}
$$




$$
\begin{aligned}
& A_{4}=-\frac{\pi\left(m^{2} \pi^{2}+n^{2} a^{2}-a^{2}\right)}{4 a} ; \\
& A_{5}=\left(\begin{array}{l}
\frac{\pi a_{85}\left(m^{2} \pi^{2}+n^{2} a^{2}-a^{2}\right)}{4 a a_{23}}+\frac{m^{2} \pi^{3}\left(m^{2} \pi^{2}+n^{2} a^{2}-2 a^{2}\right)\left(\mathrm{e}^{a}-1\right)\left(a_{21} a_{24}+a_{84} a_{22}\right)}{a^{2}\left(4 m^{2} \pi^{2}+a^{2}\right)\left(a_{21}^{2}+a_{22}^{2}\right)}+ \\
+\frac{\pi^{2} m a_{25}}{2 a_{23}}-\frac{\pi^{2} m\left(7 m^{2} \pi^{2}-n^{2} a^{2}+4 a^{2}\right)\left(\mathrm{e}^{a}-1\right)\left(a_{21} a_{84}-a_{22} a_{24}\right)}{2 a\left(4 m^{2} \pi^{2}+a^{2}\right)\left(a_{21}^{2}+a_{22}^{2}\right)}
\end{array}\right) ; \\
& A_{6}=\frac{m^{2} \pi^{3}\left(1-\mathrm{e}^{-2 a}\right)}{8 a^{4}\left(\pi^{2} m^{2}+a^{2}\right)}\left(\begin{array}{l}
\pi^{4} m^{4} C_{13}+\pi^{2} a^{2} m^{2} n^{2}\left(C_{14}+C_{23}+2 C_{32}\right)+a^{4} n^{4} C_{24}+ \\
+\pi^{2} a^{2} m^{2}\left(C_{13}-C_{14}+C_{23}+C_{24}\right)+ \\
+a^{4} n^{2}\left(C_{14}-3 C_{23}-2 C_{24}+2 C_{32}\right)+a^{4}\left(C_{23}+C_{24}-C_{14}\right)
\end{array}\right)+ \\
& +\frac{m^{2} \pi^{3}\left(-1+\mathrm{e}^{-a}\right)\left(a_{21} a_{24}+a_{84} a_{22}\right)}{2 a^{4}\left(4 \pi^{2} m^{2}+a^{2}\right)\left(a_{21}{ }^{2}+a_{22}{ }^{2}\right)}\left(\begin{array}{l}
2 \pi^{4} m^{4} C_{12}+2 a^{4} n^{4} C_{21}+2 \pi^{2} a^{2} m^{2} n^{2}\left(C_{11}+C_{22}-2 C_{31}\right) \\
+\pi^{2} a^{2} m^{2}\left(7 C_{22}-7 C_{11}-14 C_{12}+2 C_{21}\right) \\
+a^{4} n^{2}\left(8 C_{31}-C_{11}-4 C_{21}-7 C_{22}\right)+2 a^{4}\left(C_{11}+C_{12}-C_{21}-C_{22}\right)
\end{array}\right)+ \\
& -\frac{m^{2} \pi^{3}\left(1-\mathrm{e}^{-2 a}\right)\left(\begin{array}{l}
\pi^{4} m^{4} C_{12}+a^{4} n^{4} C_{21}+\pi^{2} a^{2} m^{2} n^{2}\left(C_{11}+C_{22}-2 C_{31}\right) \\
+\pi^{2} a^{2} m^{2}\left(C_{12}+C_{21}+C_{22}-C_{11}\right) \\
+a^{4} n^{2}\left(C_{11}-2 C_{21}-C_{22}\right)+a^{4}\left(C_{21}+C_{22}-C_{11}\right)
\end{array}\right) a_{85}}{8 a^{4}\left(\pi^{2} m^{2}+a^{2}\right) a_{23}}+ \\
& +\frac{-\pi^{2} m\left(1-\mathrm{e}^{-2 a}\right) a_{25}}{8 a^{3}\left(\pi^{2} m^{2}+a^{2}\right) a_{23}}\left(\begin{array}{l}
\pi^{4} m^{4}\left(C_{11}+C_{12}-C_{22}\right)+2 \pi^{2} a^{2} m^{2} n^{2}\left(C_{22}-C_{31}\right) \\
+a^{4} C_{21}\left(n^{4}+1\right)+\pi^{2} a^{2} m^{2}\left(C_{11}+C_{12}+C_{21}-C_{22}\right)-2 a^{4} n^{2} C_{21}
\end{array}\right)+ \\
& -\frac{\pi^{2} m\left(-1+\mathrm{e}^{-a}\right)\left(a_{21} a_{84}-a_{22} a_{24}\right)\left(\begin{array}{l}
\pi^{4} m^{4}\left(2 C_{11}+9 C_{12}-2 C_{22}\right)+\pi^{2} a^{2} m^{2} n^{2}\left(3 C_{11}+7 C_{22}-10 C_{31}\right) \\
+\pi^{2} a^{2} m^{2}\left(7 C_{22}-7 C_{11}-9 C_{12}+5 C_{21}\right) \\
+2 a^{4} n^{2}\left(C_{31}-C_{21}-C_{22}\right)+a^{4} n^{4} C_{21}
\end{array}\right)}{2 a^{3}\left(4 \pi^{2} m^{2}+a^{2}\right)\left(a_{21}{ }^{2}+a_{22}{ }^{2}\right)} ; \\
& A_{7}=\frac{4 \pi a m\left(\mathrm{e}^{a}+1\right)}{\left(m^{2} \pi^{2}+a^{2}\right) n} \\
& B_{1}=\frac{\pi^{3} m^{2}\left(\mathrm{e}^{2 a}-1\right)}{8\left(a^{2}+m^{2} \pi^{2}\right)} ; B_{2}=\frac{\pi\left[a^{4}\left(n^{2}-1\right)+m^{2} \pi^{2}\left(m^{2} \pi^{2}+n^{2} a^{2}\right)\right]}{4 a r_{0}^{2}\left(a^{2}+m^{2} \pi^{2}\right)} \\
& B_{3}=\frac{-2 a m \pi\left(\mathrm{e}^{a}+1\right)}{\left(a^{2}+m^{2} \pi^{2}\right) n} ; B_{4}=\frac{\rho_{1} \pi m^{2} \pi^{2}\left(-\mathrm{e}^{2 a}+1\right)}{a^{2}+m^{2} \pi^{2}} .
\end{aligned}
$$

